\title{
ㄷำ1
}

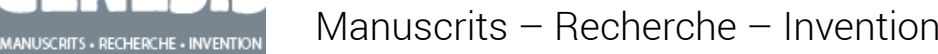

$37 \mid 2013$

Verbal - Non verbal

\section{Dossier Antonio Tabucchi}

Sandra Teroni

\section{(2) OpenEdition}

Journals

Édition électronique

URL : http://journals.openedition.org/genesis/1242

DOI : 10.4000/genesis. 1242

ISSN : 2268-1590

\section{Éditeur :}

Presses universitaires de Paris Sorbonne (PUPS), Société internationale de génétique artistique littéraire et scientifique (SIGALES)

\section{Édition imprimée}

Date de publication : 15 décembre 2013

Pagination : 189-191

ISBN : 9782840509196

ISSN : 1167-5101

\section{Référence électronique}

Sandra Teroni, « Dossier Antonio Tabucchi », Genesis [En ligne], 37 | 2013, mis en ligne le 21 mars

2016, consulté le 21 janvier 2021. URL : http://journals.openedition.org/genesis/1242 ; DOI : https:// doi.org/10.4000/genesis. 1242 


\section{Dossier Antonio Tabucchi}

Nous présentons ci-après, comme un ultime hommage à Antonio Tabucchi (1943-2012), deux documents précieux, d'une part un fragment inédit d'un projet inachevé, d'autre part la traduction d'un entretien que Tabucchi a accordé en 2004 à Sandra Teroni à l'occasion de la parution de Tristano meurt. Nous remercions chaleureusement Maria José de Lancastre, sa veuve, de nous avoir laissé la primeur de publier ce dernier inédit et d'avoir autorisé la reproduction des manuscrits de Tristano meurt qui viennent illustrer l'entretien.

Nous remercions également notre amie Sandra Teroni d'avoir eu l'idée de faire ce dossier et de nous avoir confié le texte de son entretien avec Tabucchi.

La Rédaction de Genesis

II piccolo gobbo

Inédit présenté par Sandra Teroni

L'écrivain italien Antonio Tabucchi est décédé à Lisbonne le 25 mars 2012. Sa veuve, Maria José de Lancastre, a pris la décision de donner à la BnF les papiers laissés par son mari. «C'est l'institution », déclare-t-elle, « qui leur assure le mieux les conditions nécessaires de sûreté, d'attention et d'accessibilité pour les chercheurs et c'est là que mon mari aurait aimé qu'ils soient. » L'ensemble réunit les manuscrits des romans, couchés sur des cahiers d'écolier à couverture noire, des inédits, mais aussi des lettres et des notes, dispersées entre Paris, Pise et Lisbonne.

Le dernier projet auquel travaillait Antonio Tabucchi était « un petit conte » sur Walter Benjamin. Le titre qu'il lui avait donné, Il piccolo gobbo («Le petit bossu»), renvoie à l'image sur laquelle se clôt le récit d'Enfance berlinoise de Walter Benjamin et dont Hannah Arendt, dans sa biographie de Benjamin, fait la métaphore d'une vie malheureuse. Les deux livres se côtoyaient sur la table de travail de Tabucchi, Infanzia berlinese dans la nouvelle édition réalisée à partir du dernier tapuscrit découvert à la $\mathrm{BnF}$ par Giorgio Agamben parmi les manuscrits que Benjamin, au moment de quitter Paris en 1940, avait confiés à Georges Bataille.

De ce projet que la maladie et la mort ont empêché Tabucchi de mener à son terme, restent deux fragments, de premier jet : l'un de sa main, que l'on peut dater de l'été 2011, l'autre dicté à sa femme en février 2012, un mois avant sa disparition. Il avait l'habitude de dicter certains de ses textes après les avoir « écrits mentalement ", comme il l'explique dans l'entretien qui suit, à propos de Tristano meurt ; ailleurs, il se définissait comme « un ruminant », à la manière de Flaubert, qu'il aimait par-dessus tous les grands romanciers français. C'est ce deuxième et ultime fragment inédit que nous publions ici, dans la dactylographie établie par Maria José de Lancastre, et accompagné de la traduction en français de Bernard Comment. 
- Ma lei chi è?

- Immagino che si sia chiesto perché con la Biblioteca praticamente vuota sia venuto a sedermi proprio accanto a lei.

Ben, colto di sorpresa guardò il suo vicino. Evidentemente non si era neppure accorto della sua presenza. Era un ometto dall'aspetto buffo, vestito in maniera stravagante, con un completo di tweed a grossi quadri blu e una bombetta foderata di tela cerata che aveva posato sul tavolo e sulla quale disegnava con l'indice dei ghirigori con aria affettuosa, come se l'accarezzasse.

- Mi scusi, disse Ben con aria confusa, sinceramente non l'avevo notata, ero immerso nella lettura di questo libro, e poi...

- ... e poi lei è un uomo distratto, l'uomo più distratto del mondo, solo chi la conosce può sapere fino a che punto.

- Ma lei chi è, scusi?, chiese Ben.

L'uomo si alzò, assunse per qualche secondo una posizione rigida come se si mettesse fosse sull'attenti, si risedette, dette un buffetto affettuoso alla sua bombetta come se lo desse a se stesso. - I distratti... sapesse quanti ne abbiamo nel nostro catalogo... Di solito genialità e distrazione vanno di pari passo, i distratti meritano il paradiso, C'aveva mai pensato? Non sto parlando in senso teologico, è ovvio, non mi prenda alla lettera.

- Ma certo che no, disse Ben mettendosi a ridere, era l'ultima cosa che pensavo. E ora se non le spiace vorrei finire la lettura, fra poco la Biblioteca chiude.

L'uomo alla parola "biblioteca" fece un gesto di noncuranza, come se la Biblioteca di Berlino Bibliothèque Nationale de France non gli interessasse proprio. - L'importante è che io sia riuscito a presentarmi, disse, lei è così sfuggente, quasi imprendibile. Uno come lei dovrebbe essere metodico, puntuale e , e invece arriva quando le pare, non ha orari, a volte non si vede per settimane, insomma non è stato facile, ma ci sono riuscito.

Ben lo guardava con curiosità. Chiuse il volume, posò il lapis sul quaderno con l'aria di chi rinuncia/desiste.

- Bravo!, esclamò l'ometto, finalmente! Guardi, mi era venuto il mal di pancia nel vedere davanti a lei due libri come le poesie di Baudelaire e gli scritti marxisti di Adorno. Ma come può una persona come lei che riesce a capire fin nei particolari più veri e dolorosi certe cose che solo Baudelaire è riuscito a sfiorare con un reagente di sua invenzione che viene dall'Inferno ma anche dalla polvere cosmica che tutto avvolge, come fa lei, rara creatura che sa davvero cos'è lo spleen, che ne beve un calice per ogni pagina che scrive, che ne respira una boccata per ogni occhiata che butta sul mondo, come fa a leggere con Baudelaire le saccenti paginette, dal tono professorale e presuntuoso, di questo soi-disant marxista che più marxista di lui al mondo non ce n'è?

Ben 10 guardava con attenzione. Ora l'aria L'aria di curiosità di Ben si era trasformata in una stupefazione infantile. Raramente, nei momenti più forti di estraneità da se stesso, le sue labbra carnose si allontanavano distaccavano l'una dall'altra creando sul suo volto quell'espressione di bambino che sta sognando da sveglio; ma ora quell'espressione congenita era emersa tutta, era uno sbigottimento.

- Ma lei chi è?, è mezz'ora che parla, che si dichiara felice di avermi conosciuto, ma non ci conosciamo affatto, lei non mi conosce e io non la conosco.

- Io la conosco come nessun altri, illustre Professor Benjamin. Sul mio registro che non è un registro qualsiasi, ma il Registro dei Registri, lei occupa un posto d'onore, e oggi ancora di più perché vi ho aggiunto quest'Angelo handicappato fatto di grazia e di incertezza che si è perso e non ha la forza di ritrovarsi, perché è il perdersi la sua natura.

L'uomo si alzò di nuovo di scatto in modo meccanico, si portò una mano sul petto, fece una riverenza e disse - Permetta che mi presenti: io sono il Direttore della Biblioteca Universale, la Biblioteca che raccoglie tutto ciò che l'Universo ha detto e dice, capisco che questa definizione possa sollevare le sue perplessità, ma se l'avesse letta in una pagina del suo ammirato Jorge Luís Borges le sarebbe sembrata perfettamente plausibile. Io ho molte cose da chiederle, Professore, e verrò nuovamente a visitarla, in certi particolari momenti in cui la sua persona è resa più disponibile dalle considerazioni sull'hashish riservate al suo amico Baudelaire.

si alzò Prese la sua bombetta, accennò un leggero saluto con la testa e si diresse verso l'uscita. Ad un certo punto si fermò e disse a bassa voce ma in modo ben udibile: -Mi chiamo Archibold. Mi chiami pure Archibold. Naturalmente è un nome di invenzione, ma basta saperlo entrambi.

[nella pagina seguente, con l'indicazione "da inserire"]

- Ma lei da dove viene?

- Vengo da Auschwitz. È un luogo in cui mi hanno fluidificato. Per questo ho assunto il compito di Direttore della Biblioteca dell'Universo. 


\section{- Mais vous, qui êtes-vous?}

- J'imagine que vous vous êtes demandé pourquoi je suis venu m'asseoir justement à côté de vous alors que la Bibliothèque est pratiquement vide.

Ben, surpris, regarda son voisin. Il ne s'était évidemment pas même rendu compte de sa présence. C'était un petit homme au drôle d'aspect, vêtu de manière extravagante, avec un complet de tweed à gros carreaux bleus et un chapeau melon doublé de toile cirée qu'il avait posé sur la table et sur lequel il faisait dessinait avec son index des gribouillis d'un air affectueux, comme s'il le caressait.

- Excusez-moi, dit Ben d'un air confus, je n'avais sincèrement pas prêté attention à vous, j’étais plongé dans la lecture de ce livre, et puis...

- ... et puis vous êtes un homme distrait, l'homme le plus distrait du monde, seuls ceux qui vous connaissent peuvent savoir à quel point.

- Mais vous, excusez-moi, qui êtes-vous ?, demanda Ben.

L'homme se leva, prit pendant quelques secondes une position rigide comme s'il craignait quelque chose, se rassit, donna une chiquenaude affectueuse à son chapeau melon comme s'il se la donnait à lui-même. - Des distraits... si vous saviez combien nous en avons dans notre catalogue... Habituellement le génie et la distraction vont de pair, les distraits méritent le paradis, vous n’y aviez jamais pensé ? Je ne parle pas en termes théologiques, bien sûr, ne me prenez pas à la lettre.

- Mais bien sûr que non, dit Ben en riant. C'est la dernière chose à laquelle j'aurais pensé. Et maintenant, si ça ne vous dérange pas, j'aimerais terminer ma lecture, la bibliothèque va bientôt fermer.

L'homme, au mot Bibliothèque, fit un geste d'indifférence, comme si la Bibliothèque de Berlin Bibliothèque Nationale de France ne l'intéressait aucunement. - L'important est que j'ai réussi à me présenter, vous êtes tellement fuyant, dit-il, presque insaisissable. Quelqu'un comme vous devrait être méthodique, ponctuel, et [un blanc], et au lieu de ça vous arrivez quand bon vous semble, vous n'avez pas d'horaires, parfois on ne vous voit pas pendant des semaines, bref ça n'a pas été facile, mais j'y suis parvenu.

Ben le regardait avec curiosité. Il ferma le volume, posa son crayon sur le cahier avec l'air de celui qui renonce / abandonne.

- Bravo !, s'exclama le petit homme, finalement ! Voyez-vous, ça finissait par me donner mal au ventre de voir devant vous deux livres comme les poésies de Baudelaire et les écrits marxistes d'Adorno. Mais comment une personne comme vous, qui réussit à comprendre jusque dans les détails les plus vrais et les plus douloureux certaines choses que seul Baudelaire est parvenu à effleurer avec un réactif de son invention qui vient de l'Enfer mais aussi de la poussière cosmique qui enveloppe tout, comment faites-vous, rare créature qui sait vraiment ce qu'est le spleen, qui en boit le calice pour chaque page qu'il écrit, qui en respire une bouffée pour chaque coup d'œil qu'il jette sur le monde, comment faites-vous pour lire en même temps que Baudelaire les petites pages ennuyeuses pédantes, au ton professoral et présomptueux, de ce soi-disant marxiste plus marxiste que quiconque au monde ?

Ben le regardait ave attention. Maintenant l'air L'air de curiosité de Ben s'était transformé en une stupéfaction enfantine. À de rares occasions, dans les plus forts moments d'étrangeté à soi-même, ses lèvres charnues s'éloignaient se détachaient l'une de l'autre en produisant sur son visage cette expression d'enfant en train de rêver tout éveillé ; mais à présent cette expression congénitale apparaissait entièrement, c'était une stupeur.

- Mais qui êtes-vous ?, ça fait une demi-heure que vous parlez, que vous vous prétendez heureux d'avoir fait ma connaissance, mais de fait nous ne nous connaissons pas, vous ne me connaissez pas et je ne vous connais pas.

- Je vous connais comme personne d'autre, illustre Professeur Benjamin. Dans mon registre, qui n'est pas n'importe quel registre mais le Registre des Registres, vous occupez une place d'honneur et aujourd'hui encore plus puisque je vous ai ajouté cet Ange handicapé fait de grâce et d'incertitude qui s'est perdu et n'a pas la force de se retrouver, car se perdre est sa nature. L'homme se leva à nouveau brusquement, de façon mécanique, il posa une main sur sa poitrine, fit une révérence et dit :

- Permettez que je me présente. Je suis le Directeur de la Bibliothèque Universelle, la bibliothèque qui recueille tout ce que l'Univers a dit et continue de dire, je comprends que cette définition puisse susciter votre perplexité, mais si vous l'aviez lue dans une page de ce Jorge Luis Borges que vous admirez tant, elle vous aurait paru parfaitement plausible. J'ai beaucoup de choses à vous demander, Professeur, et je viendrai à nouveau vous rendre visite, dans certains moments particuliers où votre personne est rendue plus disponible par les considérations sur le haschich réservées à votre ami Baudelaire.

Il se leva prit son chapeau melon, esquissa un discret salut de la tête et se dirigea vers la sortie. À un certain point il s'arrêta et dit à voix basse mais de façon parfaitement audible : - Je m'appelle Archibold. Vous pouvez m'appeler Archibold. Naturellement c'est un nom inventé, mais il suffit que nous le sachions tous les deux.

[page suivante, avec l'indication «à insérer»]

- Mais vous, d'où venez-vous ?

- Je viens d'Auschwitz. C'est un lieu où ils m'ont fluidifié. Voilà pourquoi j'occupe la fonction de Directeur de la Bibliothèque de l'Univers. 